\title{
Some Changes in Double-Bond Structure During the Vulcanization of Natural Rubber ${ }^{1}$
}

\author{
Frederic J. Linnig, Edwin J. Parks, and James E. Stewart ${ }^{2}$
}

(April 29, 1964)

\begin{abstract}
Near-infrared studies indicate the absence or near-absence of isolated cis and terminal double bonds in rubber vulcanized either with or without accelerators, as well as in the squalene-sulfur reaction product. The use of purified natural rubber indicates a new absorption near $6.1 \mu$ on reaction with sulfur, either with or without accelerators in the rubber system. This new absorption may be due either to a double bond with an attached sulfur atom or to conjugated double-bond systems that do not react readily with maleic anhydride. Ultraviolet spectra of chromatographic fractions show that squalene that has reacted with 15 percent of sulfur contains conjugated trienes, tetraenes, and possibly dienes, hexaenes, and heptaenes. The conjugated dienes, trienes, and tetraenes are probably present in sufficient proportions to account for a significant portion of the band at $10.4 \mu$. There is some evidence that the trienes and tetraenes, at least, may be largely all-trans in configuration. Other contributors to the band at $10.4 \mu$ are possibly isolated, trans double bonds and heterocyclic sulfur structures along the chain.
\end{abstract}

\section{Introduction}

Evidence is accumulating that changes in doublebond structure occur during the vulcanization of natural rubber with sulfur. Sheppard and Sutherland [1] ${ }^{3}$ suggested that a band near $10.4 \mu$ in the infrared spectrum of vulcanized rubber was due to an isolated trans double bond, indicating a shift of the original double bond. Recent work in our laboratory [2] has suggested the formation of conjugated double bonds. Consistent with our results, allo-ocimene, predominantly the trans- $\mathrm{C}_{4}$, cis- $\mathrm{C}_{6}$ isomer, a conjugated triene, has been isolated by Bateman et al. [3] from the reaction product of dihydromyrcene and sulfur. These authors also advanced evidence of other changes in double-bond structure [3].

The presence of conjugated double bonds in vulcanized rubber suggested [2] that they were, at least in part, responsible for the greater ease of oxidation of vulcanized rubber in comparison with unvulcanized rubber. Simple sulfur vulcanizates contain more conjugated double bonds and are more easily oxidized than accelerated vulcanizates. "Sulfurless" vulcanizates prepared with tetramethylthiuram disulfide [bis(dimethylthiocarbamoyl) disulfide] show no evidence of conjugation and are more stable than accelerated vulcanizates. The "sulfurless" and accelerated vulcanizates may, however, be somewhat stabilized by end products of the vulcanization. Other investigators [4] have related the differences in stability among these vulcanizates to differences in the assumed nature of the cross links.

\footnotetext{
1 This work was presented at the joint spring meet ing of the American Chemical Society, Division of Rubber Chemistry and The Chemical Institute of Canada, Toronto, Canada, May, 1963, and in part, at the Pittsburgh Conference on Analytical Chemistry and Applied Spectroscopy, Pittsburgh, Pa., March 1962. ${ }_{2}^{2}$ Beckman Instruments, Inc., Fullerton, Calif.

3 Figures in brackets indicate the literature references at the end of this paper.
}

The $10.4-\mu$ band, if actually due to an isolated trans double bond, could indicate a 1,4 system which has been reported to be even more reactive towards oxygen than conjugated systems when attack is at the methylene carbon atom between the double bonds [5]. The presence of the $10.4-\mu$ band in sulfur and accelerated vulcanizates and its absence in sulfurless vulcanizates are also consistent with the observed relative ease of oxidation of these vulcanizates.

Bateman et al. [3] indicated the presence of the 1,4 system among typical structures for cross-linked dihydromyrcene. These authors [3] also showed the presence of saturated structures and of ring structures containing a shifted double bond, but no adjacent original double bond and, hence, no 1,4 system.

The purpose of this paper is to examine more closely the possible changes in double-bond structure that occur during vulcanization.

\section{Materials}

(1) Natural rubber: NBS Standard Sample No. $385 \mathrm{a}$.

(2) Highly purified natural rubber: No. 2103a obtained from United States Rubber Company.

(3) Synthetic cis-polyisoprene: "Coral" rubber made by Firestone Tire and Rubber Company.

(4) Squalene: (a) 98 percent, passed through silica gel, collected under nitrogen and stored under refrigeration. (b) 90 percent, passed through a column of silica gel; the best fractions were combined and an attempt was made at further purification by growing a single crystal, but, since the crystal was colored, the extent of purification was unknown. This sample also was stored under nitrogen and under refrigeration. 


\section{Experimental Procedures}

\subsection{Sample Preparation}

(1) Films of rubber were prepared by pressing the milled sample between two sheets of aluminum foil at $125{ }^{\circ} \mathrm{C}$ for about $1 \mathrm{~min}$, using a pressure sufficient to give a film of suitable thickness, i.e., between 0.05 and $0.12 \mathrm{~mm}$ for infrared studies; three or four thicknesses were used for near-infrared studies. In general, accelerated stocks were vulcanized after the initial pressing for $24 \mathrm{hr}$, and unaccelerated stocks for $72 \mathrm{hr}$ at $125{ }^{\circ} \mathrm{C}$. Unless otherwise stated, all sulfur compounds contained 15 percent of NBS Standard Compounding Sulfur \#371c, and accelerated compounds contained an additional 7 percent of accelerator.

(2) One squalene-sulfur reaction product was prepared as follows: Purified squalene 4(a) $(8.44 \mathrm{~g})$ and sulfur $(1.52 \mathrm{~g})$, purified by the method of Murphy, Clabaugh, and Gilchrist [6], were degassed five or six times, covered with about 0.7 atm of nitrogen, sealed in a glass container, and tumbled in an oven for $23 \mathrm{hr}$. Because the oven was vented, about $3 \mathrm{hr}$ was required for the temperature to reach the reaction temperature of $150{ }^{\circ} \mathrm{C}$ after the sample had been introduced. The sample was held at $150{ }^{\circ} \mathrm{C}+1.5{ }^{\circ} \mathrm{C}$ for 20 additional hours. A second squalene-sulfur reaction product was prepared in essentially the same manner with a $1.5 \mathrm{hr}$ warmup period, using purified squalene 4 (a) $(8.47 \mathrm{~g}$ ) and purified sulfur $(1.54 \mathrm{~g})$. A third squalenesulfur reaction product, from 4 (b) and 15 percent of compounding sulfur, was prepared by heating for $22.5 \mathrm{hr}$ at $150{ }^{\circ} \mathrm{C}$ while nitrogen was bubbled through the mixture to exclude oxygen; this reaction product was used in the falling-film molecular distillation and in other studies.

\subsection{Sample Treatment}

(1) The first mentioned squalene-sulfur reaction product was separated chromatographically into 20 fractions, using a 50-50 (by weight) mixture of activated magnesia and diatomaceous earth [7]. The column was $20 \mathrm{~cm}$ long and packed in an $8-\mathrm{mm}$ i.d. tube. Ten milligrams of the reaction product in $0.1 \mathrm{ml}$ of spectrograde cyclohexane was added to the top of the column. After the material had been allowed to remain on the top of the column for a few minutes, the chromatogram was developed by adding about $14 \mathrm{ml}$ of cyclohexane through the column, with intermittent suction, over a period of about $2.5 \mathrm{hr}$. The passage of the material down the column was followed by occasionally observing the fluorescence of the various fractions under an ultraviolet lamp. The fluorescent fractions progressed about $3.5 \mathrm{~cm}$ down the column. The remainder of the column and the material that passed through the column did not fluoresce. The upper part of the column was separated in terms of the six different, fluorescing zones (brown, tan, blue, white, blue, and yellowgreen), and the remainder was cut into 13 arbitrary fractions, each $1.25 \mathrm{~cm}$ in length. The material passing through under these conditions was the 20th fraction. Each of the 20 fractions was dissolved in 3:1 cyclohexane-absolute ethanol and diluted to $5 \mathrm{ml}$. The absolute ethanol used was shown by its ultraviolet spectrum to have a low content of benzene. These solutions were diluted as much as 5:1 where necessary, and spectra of them were obtained between 210 and 500 or $600 \mathrm{~m} \mu$.

(2) The third squalene-sulfur reaction product was separated on a falling-film molecular distillation column at the temperature of boiling xylene $\left(137-140{ }^{\circ} \mathrm{C}\right)$. Two distillate fractions were taken at different points along the column.

(3) (a) The second squalene-sulfur reaction product was reacted with maleic anhydride (Fisher certified reagent) as follows:

\begin{tabular}{|c|c|c|c|c|}
\hline $\begin{array}{c}\text { Sample } \\
\text { No. }\end{array}$ & $\begin{array}{l}\text { Reac- } \\
\text { tion } \\
\text { prod- } \\
\text { uct }(g)\end{array}$ & $\begin{array}{l}\text { Maleic } \\
\text { anhy- } \\
\text { dride } \\
(\mathrm{g})\end{array}$ & $\begin{array}{l}\text { Percent } \\
\text { required, } \\
\text { maleic } \\
\text { anhydride }\end{array}$ & $\begin{array}{c}\text { Reac- } \\
\text { tion } \\
\text { tem- } \\
\text { perature }\end{array}$ \\
\hline $\begin{array}{l}1 \\
2 \\
3 \\
4 \\
5 \\
5 \\
6\end{array}$ & $\begin{array}{r}\text { 0. } 2494 \\
.2266 \\
.1999 \\
.2559 \\
.2337 \\
.2874\end{array}$ & $\begin{array}{r}0.0326 \\
.0677 \\
.0326 \\
.0642\end{array}$ & $\begin{array}{r}96 \\
218 \\
\text { None } \\
93 \\
201 \\
\text { None }\end{array}$ & $\begin{array}{l}100 \\
100 \\
100 \\
150 \\
150 \\
150\end{array}$ \\
\hline
\end{tabular}

The percent of maleic anhydride required was based on the calculated percent of molecules containing conjugated dienes, trienes, and tetraenes, as determined from curve A in figure 5 and given in table 2. In all cases the reaction time was $5 \mathrm{hr}$. After reaction, it was possible to remove some unreacted maleic anhydride by heating under a vacuum in a hot-water bath.

(b) A film of NBS Standard Sample 385a, natural rubber, was vulcanized with 15 percent of sulfur for $20 \mathrm{hr}$ at $150{ }^{\circ} \mathrm{C}$. This film was put in a glass tube containing sufficient maleic anhydride to cover the film. The tube was evacuated, heated with a water bath above the melting point of the maleic anhydride, and degassed several times. The tube was sealed off, and the maleic anhydride was reacted with the vulcanized rubber for $70 \mathrm{hr}$ at $100{ }^{\circ} \mathrm{C}$. After reaction, the film was removed from the tube and rinsed several times with acetone.

\subsection{Instruments Used}

(1) Infrared spectra were obtained with a PerkinElmer Spectrophotometer Model 221, a gratingprism instrument, with the Model 21, and with the Infracord Model 137, all equipped with $\mathrm{NaCl}$ prisms.

(2) Near-infrared, ultraviolet, and visible spectra were obtained with a Beckman DK-1 spectrophotometer. 


\section{Results}

\subsection{Synopsis of Earlier Work}

The following information was obtained in an earlier infrared study [2] of vulcanized rubber and the squalene-sulfur reaction product:

(1) The band at $10.4 \mu$ was attributed to isolated, trans double bonds resulting from a shift of the original double bond.

(2) There was an increased absorption at $11.25 \mu$ in the squalene-sulfur reaction product and not in vulcanized rubber. This absorption is probably related to terminal double bonds of the vinylidene type.

(3) New, small bands between 14 and $15 \mu$ were related to skeletal vibrations, to $\mathrm{C}-\mathrm{S}$ linkages, or to the hydrogen atoms on isolated, cis double bonds. It would appear that, if trans double bonds are formed as a result of a double-bond shift, cis double bonds should also be formed.

(4) A newly observed band at $6.25 \mu$ that could be removed by reaction for $70 \mathrm{hr}$ at $100{ }^{\circ} \mathrm{C}$ with maleic anhydride (done for the squalene-sulfur reaction product only) was related, therefore, to conjugated double bonds. Maleic anhydride reacts with conjugated double bonds to remove the conjugation.

(5) There was the appearance of an increased absorption near $6.1 \mu$, relative to the band at $6.0 \mu$, which could, however, be the result of a decreased absorption at $6.0 \mu$.

(6) The absorption near $6.1 \mu$ in the original, unvulcanized material was found to result largely from an impurity.

In this and the following section, a number of points mentioned above will be further investigated and discussed.

\subsection{Infrared Spectra}

a. New Band Near $6.1 \mu$

Squalene and purified rubber, without definite absorptions near $6.1 \mu$, were used to determine whether a new band is produced here on reaction with sulfur.

As may be seen from figure 1, the band in the spectrum of the first squalene-sulfur reaction product at $6.10 \mu\left(1639 \mathrm{~cm}^{-1}\right)$, cannot be related to anything in the spectrum of the unreacted squalene itself.

The difficulty with natural rubber is illustrated in figure 2 by the absorption at $6.15 \mu\left(1626 \mathrm{~cm}^{-1}\right)$ in curve (A) for unvulcanized, natural rubber. This absorption, due largely to an impurity, manifests itself only as an unsymmetrical band with an irregularity on the long wavelength side in curve (B) for unvulcanized, purified rubber. The new band at $6.13 \mu\left(1631 \mathrm{~cm}^{-1}\right)$ in curve (C) for purified natural rubber vulcanized with 15 percent of sulfur cannot be related to anything in the original material in curve (B). In this case, actual measurement, using a base-line technique, shows an increased absorbance at $6.13 \mu$ and about the same absorbance near $6.0 \mu$

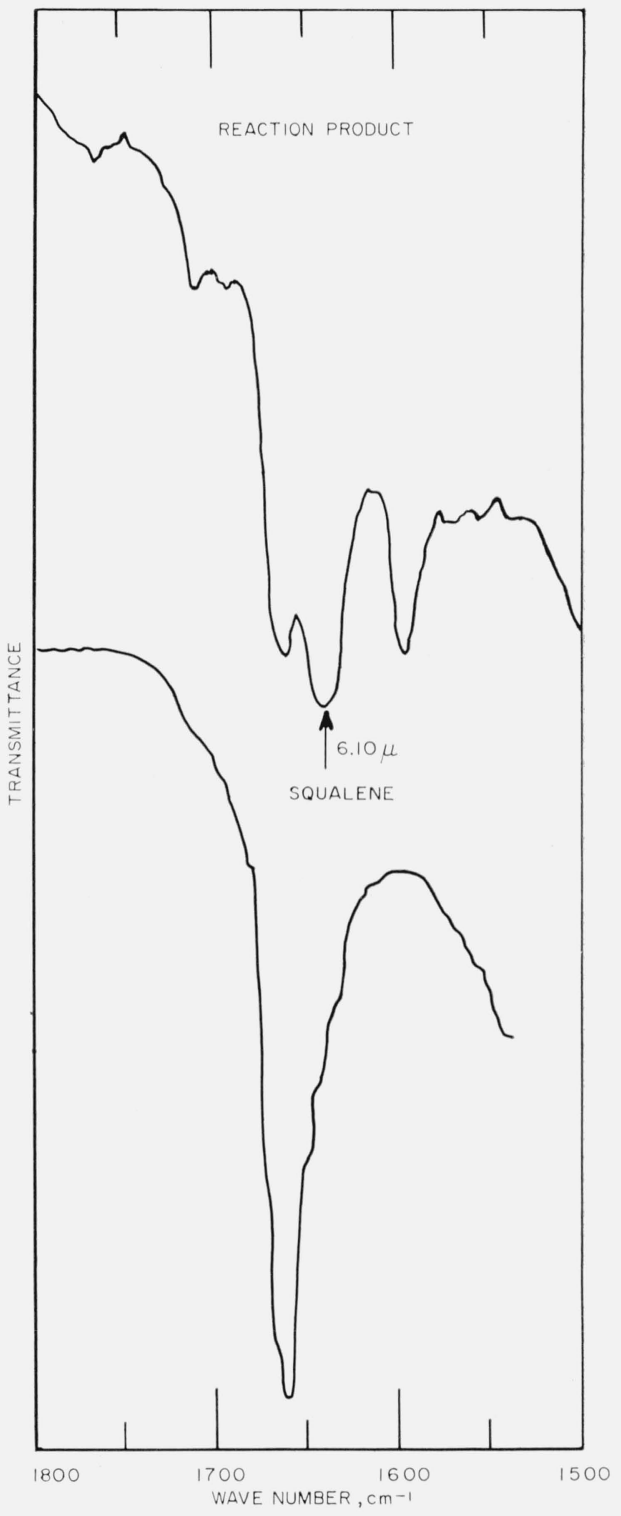

Figure 1. Infrared spectra of squalene and first squalenesulfur reaction product.

$\left(1666 \mathrm{~cm}^{-1}\right)$. Curves (D) and (E) are sulfur vulcanizates accelerated with $\mathrm{ZnDBDC}$ (zinc dibutyldithiocarbamate) and TMTD (tetramethylthiuram disulfide, NBS Standard Sample 374a), respectively. The small, sharp band at $6.13 \mu$ is no longer present, but the measured absorbance at this wavelength is about the same in curves (C), (D), and $(\mathrm{E})$. However, the absorbance at $6.0 \mu$ is definitely greater in curves (D) and (E) than in curves (B) and (C). This, of course, might cause an increased absorbance near $6.13 \mu$ through the effect of overlapping bands. On the other hand, absorbance is reduced near $6.0 \mu$ in squalene after reaction with sulfur. 


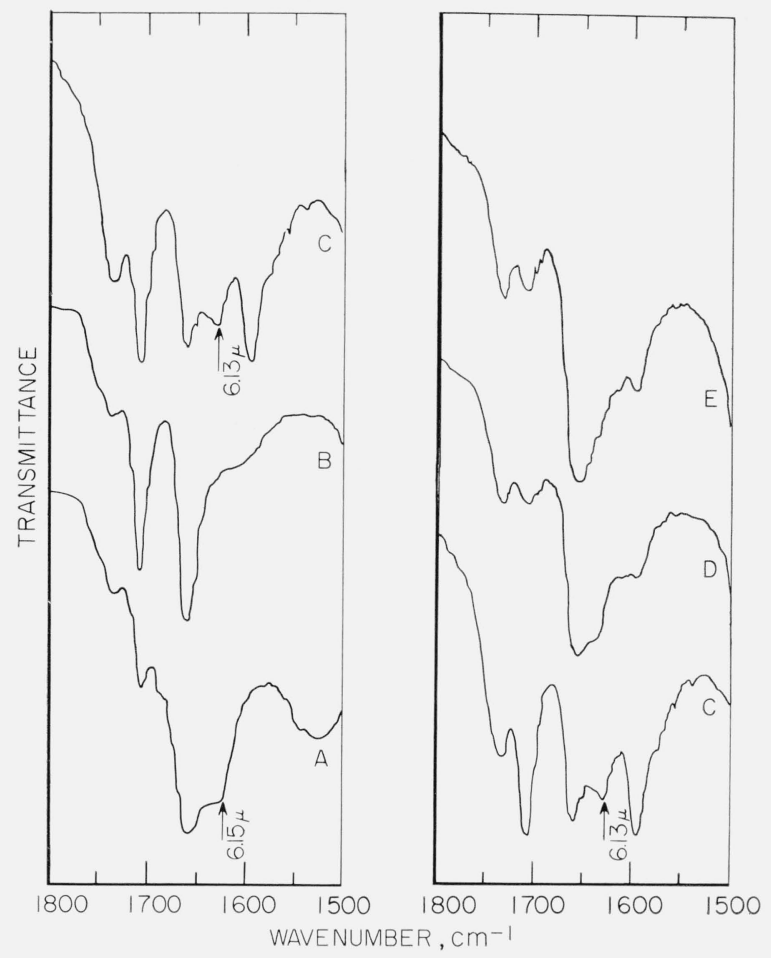

FIgURE 2. Infrared spectra of unvulcanized and vulcanized natural rubber.

A-unvulcanized NBS 385a, B-rnvolcanized prrified rubber, C-purified rubber vulcanized with 15 percent of s'lf $1 \mathrm{r}$, D-p'rified robber vulcanized with 15 percent of s?lfur and 7 percent of ZnDBDC, and E-purified rubber vulcanized with 15 percent of sulfur and 7 percent of TMTD.

Although the presence of a new band near $6.1 \mu$ is not definite in the case of sulfur-vulcanized, unpurified rubber (NBS Standard Sample 385a), approximate measurements indicate increased absorbance at both 6.0 and $6.13 \mu$. Accelerated vulcanizates of NBS 385a show an increased absorbance at $6.13 \mu$ and relatively small changes near $6.0 \mu$. The slight difference between 6.10 and $6.13 \mu$ for the position of the band in the two systems may indicate the presence of two different groups.

Thus, it now appears likely that, as a result of vulcanization, there is new or increased absorption near $6.1 \mu$, even in the case of unpurified rubber.

\section{b. Possible Interpretation of the 6.1- $\mu$ Band}

This new absorption near $6.1 \mu$ in vulcanized rubber and in the squalene-sulfur reaction product could be due to the cis double bonds or terminal double bonds mentioned earlier. Terminal double bonds of the vinylidene type, suggested by the increased absorption at $11.25 \mu$ in the squalene-sulfur reaction product, may be partly responsible for the band at $6.1 \mu$ in this case. According to Bellamy [8], trans double bonds absorb, on the average, very near $6.0 \mu$, where the original double bond absorbs, so that this structure should not be responsible for the new band. Substitution of sulfur for the hydrogen atoms on the original double bond should shift the carbon-to-carbon, double-bond stretching-fre- quency toward $6.1 \mu$ or beyond. In fact, Mikawa [9] has found that methyl vinyl sulfide absorbs at $6.31 \mu\left(1585 \mathrm{~cm}^{-1}\right)$. This type of structure is, therefore, a suggested cause of the new absorption. One other interpretation of the $6.1-\mu$ band is that it is caused by conjugated double bonds. Two isomers of allo-ocimene, the trans- $\mathrm{C}_{4}$, trans- $\mathrm{C}_{6}$ and the trans $-\mathrm{C}_{4}$, cis $-\mathrm{C}_{6}$, have been reported by $\mathrm{O}$ 'Connor and Goldblatt [10] to absorb at 6.06 and $6.08 \mu$, respectively. The trans-cis isomer was isolated from the dihydromyrcene-sulfur reaction product [3].

\section{c. Relation of New Absorptions to Cross-linking}

In an attempt to relate cross-linking to some of the new absorptions in the infrared spectra of the squalene-sulfur reaction-product, infrared spectra were obtained of the two distillate fractions and of the residue from the molecular distillation. The $6.25-\mu$ band (related to conjugation) in the residue, presumably containing the cross-linked material, was stronger than in the distillates, which contained unreacted squalene and reacted monomolecular squalene [11]. The darker color of the residue is consistent with a higher concentration of conjugated double bonds. However, when allowances are made for the dilution effect of the unreacted material, as measured by the greater intensity of the characteristic bands at 6.0 and $12.0 \mu$ in the distillates, it cannot definitely be concluded that there is a relationship between conjugation and cross-linking; it is, however, suggested. The band at $10.4 \mu$ is probably somewhat stronger in both distillates than in the residue, but the shoulder on the long-wavelength side, at about 10.5 to $10.6 \mu$, is not as pronounced in the distillates. It is in this latter region that two of the tetrahydrothiophene ring compounds found in the dihydromyrcene-sulfur reaction product have been reported to absorb [12, 13].

\section{d. Relation of Sulfur Content to the 6.25- $\mu$ Band}

It has been found that, with NBS 385a, the intensity of the band at $6.25 \mu$, related to conjugation, increases with increased sulfur content up to about 20 percent, and then decreases with larger sulfur contents, with very little remaining at 32 percent of sulfur. On the other hand, with both purified rubber and the synthetic rubber, although there may be some decrease after a maximum at about 20 percent, a strong band still remains at 32 percent. This result is, presumably, due to the effect of the nonrubber constituents in the unpurified, natural rubber. An absorption still remains near $6.1 \mu$, even in purified rubber when vulcanized with 32 percent of sulfur. A band is present at this wavelength in both unpurified rubber and synthetic rubber vulcanized with 32 percent of sulfur.

\subsection{Near-Infrared Spectra}

Near-infrared studies have aided in clarifying the nature of the bands near 6.1 and $11.25 \mu$ (in the squalene-sulfur reaction product only) and between 14 and $15 \mu$. 
It will be noted in figure 3 that the spectrum of the first squalene-sulfur reaction product has a small band near $1.64 \mu$ not exhibited in the unreacted squalene. This band is probably characteristic of terminal double bonds [14], and its presence is consistent with the increased absorption at $11.25 \mu$. A comparison of the two curves shows no evidence of a new absorption band in the lower curve, near $2.14 \mu$, characteristic of $c i s$ double bonds [14].

Curve $\mathrm{A}$ in figure 4 is of purified, natural rubber and contains no band at 1.64 or $2.14 \mu$. The absence of a band at $1.64 \mu$ has been used by Fraga $[15 \mathrm{~A}]$ to indicate the absence of 3,4 structures in natural polyisoprenes. Binder $[15 \mathrm{~B}]$ from other evidence takes a contrary view. New absorption bands do not appear at 1.64 or $2.14 \mu$ in curve B of purified rubber vulcanized with 15 percent of sulfur. Thus, the terminal double bonds in the squalene-sulfur reaction product are probably the result of end effects in the smaller molecule. There is only the barest suggestion of an absorption at 1.64 $\mu$ in curves $C$ and $D$ for vulcanizates accelerated with ZnDBDC and TMTD, respectively. Similar results are obtained when unpurified rubber (NBS 385a) is employed. Thus, except for the result of end effects in squalene, few or no cis or terminal double bonds result from reaction with sulfur, even in the presence of accelerators, and there cannot be much, if any, contribution of these structures to the band near $6.1 \mu$. Since there is no confirmatory evidence in the near-infrared of cis double bonds, the small bands between 14 and $15 \mu$ are possibly due to skeletal vibrations or to $\mathrm{C}-\mathrm{S}$ linkages.

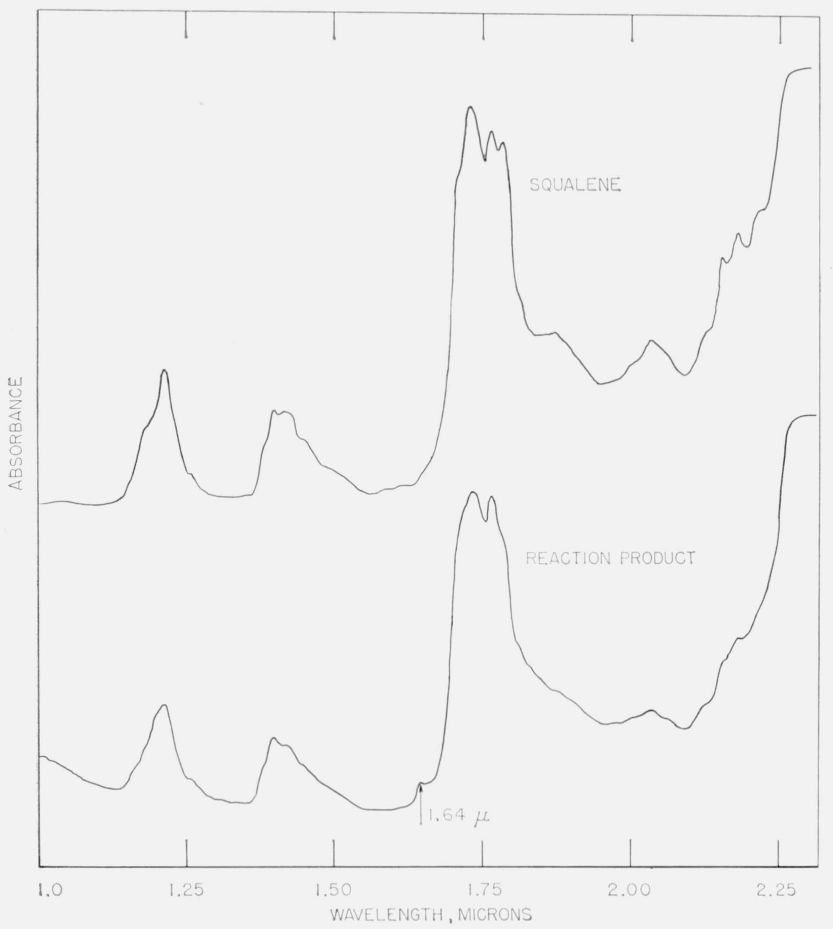

Figure 3. Near-infrared spectra of squalene and squalenesulfur reaction product: squalene, 200.4 g/liter; reaction product $203.7 \mathrm{~g} /$ liter; 1 -cm path length.

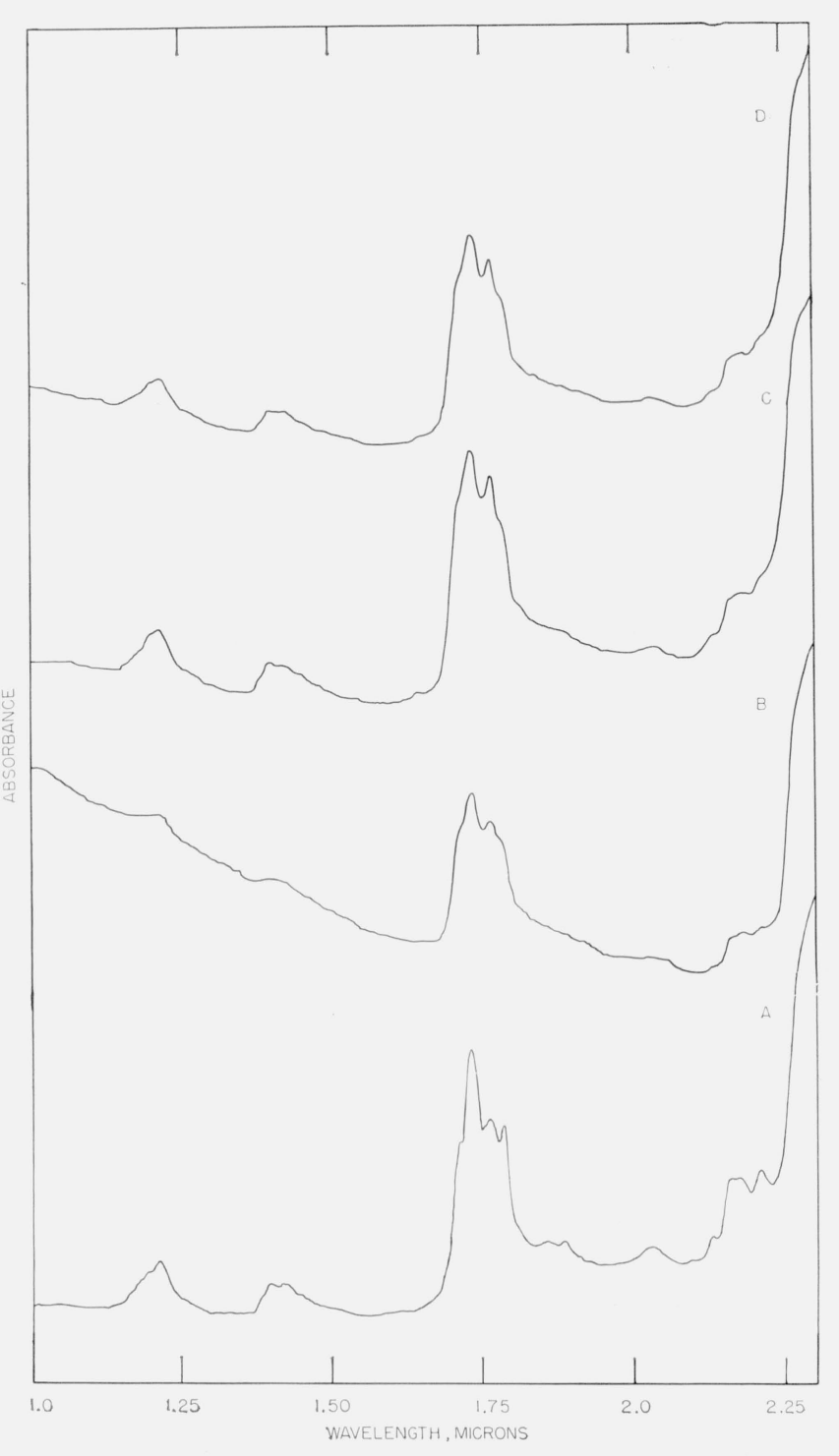

Figure 4. Near-infrared spectra of unvulcanized and vulcanized purified natural rubber.

A-unvulcanized rubber, B-rubber vuleanized with 15 percent of sultur, C-rubber vulcanized with 15 percent of sulfur and 7 percent of $\mathrm{ZnDBDC}$, and D-rubber vulcanized with 15 percent of sulfur and 7 percent of TMTD.

\subsection{Ultraviolet Spectra and Chromatography}

Vulcanized rubber and the squalene-sulfur reaction product presumably contain conjugated systems [2], free sulfur, and sulfur structures including heterocyclics, straight-chain monosulfides, and polysulfides [3]. Conjugated structures absorb in the ultraviolet, with conjugated dienes giving a single absorption, and the higher conjugated systems giving three-peak absorptions. These peaks occur at longer wavelengths with increasing numbers of conjugated double bonds in the systems [16]. Koch [17, 18], Davis [19], Minoura [20], and Baer and Carmack [21] have presented ultraviolet spectra of elemental sulfur and of a variety of sulfides and 
polysulfides. Cyclic sulfides show an increased general absorption with decrease in wave length. Straight-chain mono-, di-, and polysulfides exhibit shoulders or broad maxima at wavelengths that increase with increasing numbers of sulfur atoms. Postovskaya, Salimov, and Kuzminskiǔ [22], and Dogadkin and Pavlov [23] have used these absorptions to determine the number of sulfur atoms in sulfide cross-links of vulcanized rubber.

The second squalene-sulfur reaction product, dissolved in spectrograde cyclohexane, gave the spectrum presented in $\mathrm{A}$ of figure 5 . In the carotenoid or isoprenic series, such as might be present here, conjugated trienes are reported to absorb near 275, $\mathbf{2 8 5}$, and $297 \mathrm{~m} \mu$ and tetraenes near $305, \mathbf{3 1 8}$, and $332 \mathrm{~m} \mu$, [16], the boldface number being the strongest absorption. Similar conjugated dienes absorb near $240 \mathrm{~m} \mu$ [16]. These absorptions very closely fit the curve given in figure 5. There are also

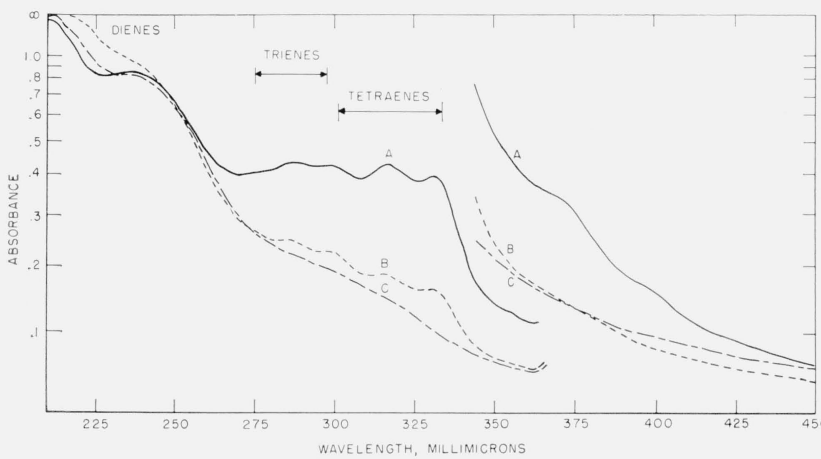

Figure 5. Ultraviolet spectra of A-squalene-sulfur reaction product (53.3 mg/liter, 210-360 mu; $0.178 \mathrm{~g} /$ liter, beyond $360 \mathrm{m \mu}$ ), B-squalene-sulfur reaction product reacted with 218 percent of maleic anhydride at $100{ }^{\circ} \mathrm{C}$ (Sample No. 1-69.0 mg/liter, 210-360 mu; $0.230 \mathrm{~g} /$ liter, beyond $360 \mathrm{m \mu}$ ), and $\mathrm{C}$ squalene-sulfur reaction product reacted with 201 percent of maleic anhydride at $150{ }^{\circ} \mathrm{C}$ (Sample No. $4-69.0 \mathrm{mg} / \mathrm{liter}$, 210-360 mu;0.230 g/liter, beyond $360 \mathrm{m \mu}$ ) at 1-cm path length.

The concentrations given for curves $\mathrm{B}$ and $\mathrm{C}$ indicate the concentration of squalene-sulfur reaction product only in the reaction mixture.

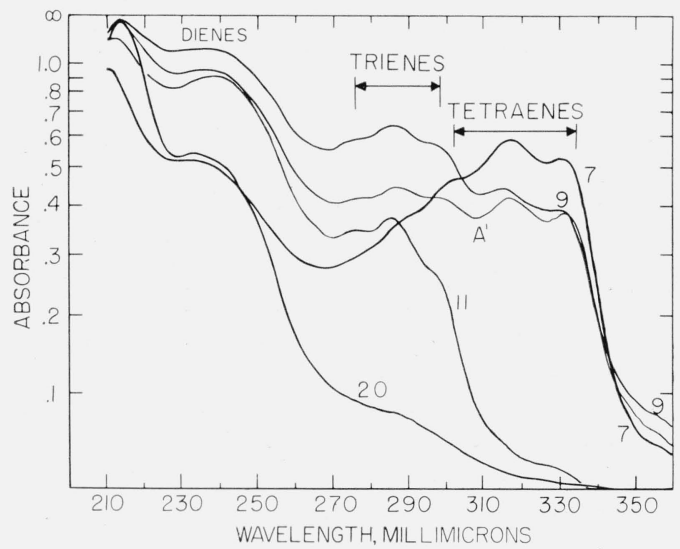

FIGURE 6. Ultraviolet spectra of the original squalene-sulfur reaction product, $A^{\prime}$, and chromatographic fractions $7,9,11$, and 20. shoulders, a slight but reproducible one at $356 \mathrm{~m} \mu$ and others at 372,398 , and 420 to $425 \mathrm{~m} \mu$ (these latter absorptions being more pronounced in the first reaction product), that could be due to conjugated hexaenes absorbing near $356, \mathbf{3 \% 6}$, and 397 $\mathrm{m} \mu$ and heptaenes absorbing near 378 400, and $425 \mathrm{~m} \mu$ [16]. There is little evidence of pentaenes or, at this concentration, of systems with more than seven conjugated double bonds.

Chromatographic separation of the first reaction product into 20 fractions yielded the four significant fractions given in figure 6 . The absorbance of the original reaction product is given by curve $A^{\prime}$. In curve 7 , reduced absorption in the triene region emphasizes the three absorptions at the wavelengths characteristic of tetraenes. On the other hand, the last two peaks characteristic of tetraenes are reduced in intensity in the curve for fraction 9 , as would be the case if the concentration of the tetraenes were somewhat lowered, the third peak remaining unchanged presumably because of the proximity of the long-wavelength peak of the trienes. In the curve for fraction 11, there is only a shoulder in the tetraene region (that could be due either to tetraenes or a polysulfide) on the side of the triene peaks which are clearly revealed. Again, in the curve for fraction 20 , there is merely a shoulder on the $236-\mathrm{m} \mu$ peak, characteristic of dienes or sulfide structures, which is also present in the spectra of the other fractions. None of the fractions contained more than shoulders in the region beyond $360 \mathrm{~m} \mu$. Thus, if hexaenes and heptaenes are actually present, they are not present in sufficient proportions to give well defined, characteristic, three-peak spectra in any of the fractions.

The ultraviolet spectrum of the first reaction product was nearly identical with that of the second. The third reaction-product was not as dark as the first two, and when it was kept in a capped bottle under nitrogen in a freezer $\left(-26{ }^{\circ} \mathrm{C}\right)$ for several years after preparation, it resembled fraction 9 in the region between 210 and $360 \mathrm{~m} \mu$. The first reaction product, after a similar period of storage, also gave a curve similar in shape to that of fraction 9, with reduced absorption in the triene and tetraene regions.

The separation of the reaction product into fractions exhibiting the three-peak absorption spectra characteristic of trienes and tetraenes is strong evidence of their presence. In view of this conclusion, it is reasonable to suppose that the peak at $236 \mathrm{~m} \mu$ is probably due, in part as least, to conjugated dienes. Analysis of the first squalene-sulfur reaction product by the method of Bartlett and Skoog [24] showed the presence of about 0.4 percent of elemental sulfur, a proportion shown not to affect the ultraviolet spectrum of the reaction product. In the qualitative interpretation, interference from sulfur structures seems most likely in the case of conjugated dienes, although part of the general absorption throughout the region studied here is probably due to sulfur structures. The Russian authors [22, 23] were able to use ultraviolet studies to estimate the number of sulfur atoms in polysulfide cross-links, probably 
because their compounds had been vulcanized for relatively short periods of time, compared to the 20 to $23 \mathrm{hr}$ used here for the squalene-sulfur reaction product. There is evidence that polysulfides tend to decompose to shorter sulfidic links on extended reaction [3, 25], whereas conjugation tends to increase with reaction time.

\subsection{Treatment With Maleic Anhydride}

\section{a. Discussion}

Maleic anhydride reacts with conjugated systems, through a Diels-Alder reaction, to remove the conjugation. The band at $6.25 \mu$ in the spectrum of the squalene-sulfur reaction product was completely removed in $70 \mathrm{hr}$ at $100{ }^{\circ} \mathrm{C}$, suggesting that it was due to conjugation; whereas the bands at 6.0 and $6.1 \mu$ remained largely unchanged, except for an apparent increase in the absorption at $6.0 \mu$ relative to $6.1 \mu$ which could be related to increased absorption in the adjacent carbonyl region. This situation suggests that the band near $6.1 \mu$ is due to some structure other than conjugated double bonds. Sterically unhindered systems, such as those containing trans-trans conjugation, react readily at this temperature. Sterically hindered systems, such as those containing cis-cis and cis-trans conjugation, are usually much more difficult to react, but are sometimes isomerized into the more reactive trans-trans form at higher temperatures [26]. There is, unfortunately, at least one exception in what is apparently a cis-trans isomer of 2,6-dimethyl-3,5-octadiene [27]. The structure of this compound is similar to structures that might be expected in the squalene-sulfur reaction product, and the compound reacts with maleic anhydride when reated in a water bath for $15 \mathrm{hr}$.

The effect of temperature on the infrared and ultraviolet spectra of the maleic anhydride reactionproduct was investigated, along with the effect of maleic anhydride on vulcanized rubber.

Preliminary work had suggested that reaction of the squalene-sulfur reaction product with maleic anhydride at $100{ }^{\circ} \mathrm{C}$ for only $5 \mathrm{hr}$ was sufficient to remove the band at $6.25 \mu$. Consequently, reactions were carried out for this period of time using the second squalene-sulfur reaction product and the indicated amounts of maleic anhydride at 100 and $150^{\circ} \mathrm{C}$, to determine whether any of the unreactive types that would not react at $100{ }^{\circ} \mathrm{C}$ could be isomerized at the higher temperature to the more reactive trans-trans isomers.

\section{b. Squalene-Sulfur Reaction Product; Infrared Spectra}

In the samples included in section 3.2, removal of the band at $6.25 \mu$ was complete in 5 hr, only at $150{ }^{\circ} \mathrm{C}$. In addition, at the higher temperature, the band at $6.01 \mu$ is reduced relative to the one at $6.10 \mu$, instead of the converse that would have been expected. There is indeed, in these cases, some additional reaction at the higher temperature, as indicated (in the ultraviolet studies to follow) by the reduced absorbance in curve $\mathrm{C}$ of figure 5 in the triene and tetraene regions. This change is probably in part related to the more complete removal of the band at $6.25 \mu$.

Possible interpretations of the small effect that is observed on the bands near 6.0 and $6.1 \mu$ are discussed below. This effect could suggest that some of the conjugated systems are absorbing near 6.0 rather than $6.1 \mu$. Shifting of double bonds in 1,4 or 1,5 systems at the higher temperature could also cause reduction in the intensity of the $6.0-\mu$ band, because the absorption at this wavelength, characteristic of the original double bond or the shifted trans double bond, could be removed. Direct reaction with maleic anhydride to remove the isolated, original, double-bond systems is highly unlikely, since uninitiated reactions related to isolated double bonds have been reported to take place only at temperatures above $150^{\circ} \mathrm{C}$ and to involve the hydrogen atoms on the $\alpha$-carbon atom instead of the double bond itself [28]. It should be noted that Binder [15B] gives evidence for the removal of the double bond, but does not give the temperature of the reaction. Pinazzi, Cheritat, and Pautrat [29] obtained a reaction product with rubber and maleic anhydride, using heat alone, in which part of the double bonds are shifted to the methyl side chain at temperatures above $180{ }^{\circ} \mathrm{C}$. If this reaction were to take place to any extent at $150{ }^{\circ} \mathrm{C}$, it could explain the relative increase in the intensity of the band near $6.1 \mu$, since the shifted terminal double bond would be expected to absorb here.

Maleic anhydride has a strong band at $14.4 \mu$. As judged by removal or reduced intensity of this band, reaction at $150{ }^{\circ} \mathrm{C}$ does reduce the amount of unreacted maleic anhydride to an unexpected extent, considering the amounts present. Treatment of the blank at 100 and $150{ }^{\circ} \mathrm{C}$, or distillation of the blank following treatment, does not change the infrared spectrum appreciably. Apparently, further reaction does not occur, and the structures causing absorption at $6.1,6.25$, and $10.4 \mu$ are not volatile.

\section{c. Squalene-Sulfur Reaction Product; Ultraviolet Spectra}

The ultraviolet absorption spectrum of the maleic anhydride reaction-product, obtained at $100{ }^{\circ} \mathrm{C}$ with 218 percent maleic anhydride (Sample No. 1) and dissolved in cyclohexane, is given in curve B of figure 5. Curve $\mathcal{C}$ in the same figure was made by using the reaction product obtained at $150{ }^{\circ} \mathrm{C}$ with 201 percent maleic anhydride (Sample No. 4), dissolved in 3:1 cyclohexane-absolute ethanol; this reaction product was not quite completely soluble. It had been shown that the curve for the squalenesulfur reaction product was identical in both solvents. Absorbance measurements given in table 1 were made at 236,287 , and $317 \mathrm{~m} \mu$, as being indicative of changes in absorbance in the diene, triene, and tetraene regions, respectively. The measurements on curves $\mathrm{B}$ and $\mathrm{C}$ were corrected for the dilution effect of the added maleic anhydride, and for the differences in concentration of the material used, in 
an effort to put them on a basis comparable with those made on curve A. Data for the other samples are not presented.

TABLE 1. Comparison of ultraviolet absorbances ${ }^{\text {a }}$

\begin{tabular}{|c|c|c|c|}
\hline \multirow{2}{*}{ Treatment with maleic anhydride } & \multicolumn{3}{|c|}{$\mathrm{m}_{\mu}$} \\
\hline & 236 & 287 & 317 \\
\hline $\begin{array}{l}\text { Untreated (Curve A)c } \\
100^{\circ} \mathrm{C} \text { (Curve B) b d } \\
150^{\circ} \mathrm{C} \text { (Curve C) b d }\end{array}$ & $\begin{array}{r}0.839 \\
.943 \\
.808\end{array}$ & $\begin{array}{r}0.430 \\
.250 \\
.218\end{array}$ & $\begin{array}{r}0.426 \\
.186 \\
.144\end{array}$ \\
\hline
\end{tabular}

a Data obtained from curves in figure 5.

b Data corrected for the dilution resulting from the addition of maleic anhydride and for differences in concentration from curve $\mathrm{A}$.

c Squalene-sulfur reaction product.

d Squalene reaction product, reacted with maloic anhydride.

In both curves B and C, there is little change in absorption in the region characteristic of dienes, but a pronounced decrease in absorption in the regions characteristic of trienes, tetraenes, hexaenes, and heptaenes. The reduced absorption and, where noted, decreased detail in curve $\mathrm{C}$ when compared to curve $\mathrm{B}$ in these regions, is presumably consistent with reduced absorption at $6.25 \mu$ in the infrared region when the reaction is carried out at $150{ }^{\circ} \mathrm{C}$.

In other tests of the first squalene-sulfur reaction product (not listed in section 3.2), in which the band at $6.25 \mu$ was removed at $100{ }^{\circ} \mathrm{C}$, there was little change in the ultraviolet absorption on going from 100 to $150{ }^{\circ} \mathrm{C}$, except for somewhat less detail in the triene and tetraene regions and a 12-16 percent reduction in absorbance in the diene region.

Decreasing the proportion of maleic anhydride caused little change in the ultraviolet spectra. At the lower concentration, there was an unexplained crossing over of the curves for the 100 - and $150{ }^{\circ} \mathrm{C}$ reaction products near $342 \mathrm{~m} \mu$ in the short wavelength section but not in the other. Distillation indicating that the structures responsible for the bands are not volatile. The only time that distillation resulted in an appreciable reduction in absorption was for the spectrum beyond $340 \mathrm{~m} \mu$ of sample 4 containing 93.1 percent of maleic anhydride and reacted at $150{ }^{\circ} \mathrm{C}$. The difference was most pronounced at the longer wavelengths.

These results offer further confirmation of the assignment of the original, pertinent, ultraviolet bands to conjugated trienes and tetraenes. There appears to be little confirmation of the presence of dienes. However, maleic anhydride shows an absorbance of 0.176 at $236 \mathrm{~m} \mu$ for $15.2 \mathrm{mg} /$ liter, corresponding to about the 200 percent level. The contribution of the maleic anhydride structure in the reaction product to the absorbance at $236 \mathrm{~m} \mu$ is not known, but, to the extent that free maleic anhydride is present, it would increase the absorbance at this wavelength. For the distilled samples, the solutions were prepared assuming that no maleic anhydride had been removed. In cases where appreciable amounts of maleic anhydride had been removed, this method of preparation would increase the actual concentration of squalene-sulfur reaction product beyond the amount intended and thus increase absorption in general, including that in the diene region. Thus, there is reason to assume some reduction in absorption in the diene region, too. The picture is further complicated, however, by the fact that interference from sulfur compounds is most likely in this region. Thus, complete support of the hypothesis is dependent on whether maleic anhydride also reacts with the sulfur compounds present to give reduced absorbance in the diene region. As an example, tetrahydrothiophene, the parent compound of the substituted materials found in the dihydromyrcene-sulfur reaction product $[12,13]$, does not react with maleic anhydride in $90 \mathrm{~min}$ at $125{ }^{\circ} \mathrm{C}$ [30]. This type of compound shows an increased general absorption at shorter wavelengths. Because of the extended reaction time, polysulfides having large numbers of sulfur atoms would not be expected to be present [3]. Since large numbers of sulfur atoms are probably not involved, reduced absorption and removal of the shoulders beyond $340 \mathrm{~m} \mu$ suggest that the shoulders are due to hexaenes and heptaenes.

The residual absorption after reaction with maleic anhydride may, of course, still be due to unreactive conjugated systems. However, reactions above 150 ${ }^{\circ} \mathrm{C}$ would not be meaningful, since this was the temperature at which the squalene-sulfur reaction product was formed. Furthermore, Bloomfield and Naylor [31-33] have indicated that polysulfides decompose, and react with olefins, at temperatures from 140 to $180{ }^{\circ} \mathrm{C}$. Another possible cause of this residual absorption is scattering.

On the other hand, the ultraviolet curve for the maleic anhydride reaction product obtained at $150^{\circ} \mathrm{C}$ is quite similar to those of some of the sulfur compounds mentioned earlier, and may, quite possibly, be due largely to this type of material. If such is, indeed, the case, the band at $6.1 \mu$ cannot, of course, be due to unreactive conjugated double bonds. The other possibility, suggested earlier, is isolated double bonds with attached sulfur atoms. In the squalene-sulfur reaction product, there is also some contribution from terminal double bonds to the band at $6.1 \mu$.

\section{d. Vulcanized Rubber; Infrared Spectrum}

Reaction of the film of vulcanized rubber with maleic anhydride removed the band at $6.25 \mu$ in the infrared spectrum, as had been found earlier for the squalene-sulfur reaction product [2].

The band reported by Mikawa [9] at 6.31 $\mu$ in methyl vinyl sulfide could cast doubt on the interpretation of the $6.25-\mu$ band as being due to conjugation, if the band at $6.31 \mu$ is removed by reaction with maleic anhydride.

\section{Discussion}

\subsection{Mechanisms}

The first formula given below shows two adjacent 
isoprene units of a rubber molecule, and the second, the trans double bond resulting from a shift of the original double bond through a free-radical mechanism, as originally proposed by Sheppard and Suther-

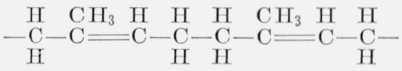

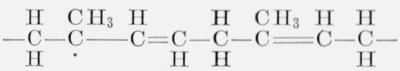

land [1]. If trans double bonds to which the 10.4- $\mu$ band has been attributed are actually present, they must be produced by a shift of the original double bond, since the introduction of a new double bond would automatically produce a cumulative or conjugated system. The new trans double bond produces a 1,4 system. Two shifts of two double bonds toward each other, or two successive shifts of one double bond, are, barring more involved mechanisms, necessary to produce the conjugated dienes suggested by the ultraviolet spectra.

On the other hand, the observed conjugated trienes could be produced by the introduction of a new double bond midway between two original double bonds. The observed conjugated tetraenes could be produced by the introduction of a new double bond at this same point, combined with two successive shifts of another original double bond toward these three. Similar reasoning applies to the more highly conjugated polyenes suggested by the ultraviolet spectra. Of course, similar numbers of conjugated double bonds could also result simply from many different shifts alone. However, the introduction of new double bonds is consistent with the work with dihydromyrcene of Bateman, Glazebrook, and Moore [3], in which they isolated, among other compounds, alloocimene, and with our work with propylene, in which we isolated propadiene (allene) [34].

In any event, regardless of whether isolated trans double bonds exist, shifts of double bonds by some mechanism are necessary for the production of some of the observed conjugated systems, e.g., conjugated dienes, tetraenes, and hexaenes, as well as other evennumbered systems. Whether longer conjugated systems would be produced, with the same proportion of sulfur, in the larger molecule, rubber, is not known.

The observed different degrees of conjugation could result from reaction at a single point, as part of a chain mechanism. They could also be related to the statistical probability of producing the observed concentration of the different systems as a result of stepwise, individual reactions at certain necessary sites with the proportion of sulfur used. This would be difficult to determine, since many sulfur atoms may go into one cross-link [25].

\subsection{Interpretation of the 10.4- $\mu$ Band}

The band at $10.4 \mu$, previously attributed to isolated trans double bonds [1, 2], presents an interesting problem. It has also been attributed by Glazebrook and Saville [13] and by Bateman, Glazebrook, Moore, and Saville [12] to the substituted tetrahydrothiophenes that were formed in their studies with dihydromyrcene. It is instructive to follow through the implications of the data presented here and in our previous paper [2], in terms of an interpretation of the $10.4-\mu$ band.

\section{a. Isolated Trans Double Bonds}

In figure 6 of the earlier paper [2], there was some residual band present at $10.4 \mu$ in the spectrum of the maleic anhydride reaction-product. Spectra of some of the other samples of this reaction product have also shown residual absorption here, indicating the presence either of unreacted conjugation (see section $5.2 \mathrm{c}$ ) or of some isolated trans double bonds and, therefore, of the 1,4 double-bond structure. The actual proportion present of structures absorbing at $10.4 \mu$ may be greater than that indicated by these spectra, because of the effect of increased absorption on both sides of the band [2] due to reaction with the maleic anhydride.

\section{b. Tetrahydrothiophene Structures}

The substituted tetrahydrothiophenes isolated in the reaction of dihydromyrcene with sulfur have been reported to absorb near 10.5 to $10.6 \mu$, with one absorbing at $10.44 \mu$, also [12, 13]. The breadth of the band at $10.4 \mu$ in the spectra of rubber and the squalene-sulfur reation product does allow for the presence of structures of the type absorbing at 10.44 $\mu$, and even for some of those absorbing between 10.5 and $10.6 \mu$. This latter situation is especially true in the spectrum of the squalene-sulfur reaction product, where there is a definite shoulder between 10.5 and $10.6 \mu$. The shoulder here, its absence from vulcanized rubber, and the isolation of tetrahydrothiophenes in the dihydromyrcene-sulfur reaction product might suggest that these structures are more likely in smaller compounds and less likely in larger molecules, such as rubber. As indicated previously [2], the band at $10.4 \mu$ in vulcanized rubber shifts toward longer wavelengths with increasing sulfur content. This result is in agreement with the findings of Sheppard and Sutherland [1]. With unpurified natural rubber, the band showed, with 32 percent of sulfur, a flat spot between $10.5 \mu$ and $10.55 \mu$, with what appeared to be a slight shoulder at $10.35 \mu$. On the other hand, purified rubber and synthetic rubber showed a maximum absorption at about $10.47 \mu$ with 32 percent of sulfur. These effects might be interpreted as resulting from an increase in tetrahydrothiophenes with increased sulfur content. The longer wavelength for the unpurified rubber could presumably also be related to the reduced amount of conjugation (near-absence of the band at $6.25 \mu$ and consequent reduction in the band at 10.35 or $10.4 \mu$; see section $5.2 \mathrm{c}$ ) in natural rubber vulcanized with 32 percent of sulfur, as compared with the amount present when purified or synthetic rubber is used. It must also be remembered that the tetrahydrothiophenes in different structural environments could absorb at wavelengths other than those reported above. 


\section{c. Conjugated Double Bonds}

Many conjugated polyenes absorb near $10.4 \mu$. Lunde and Zechmeister [35] showed that, in the case of conjugated diphenyl polyenes, at least one trans double bond had to be present to produce an appreciable band between 10.0 and $10.6 \mu$. This absorption is sometimes a single band, sometimes shifted from its usual position near $10.4 \mu$, and sometimes split into two bands. Unfortunately, it is difficult to predict with certainty, from theory, the types of conjugated systems that would or would not lead to shifting or splitting of this band. Some pertinent experimental evidence on this point follows:

(1) Conjugated cis-trans systems of the $-\mathrm{CH}=\mathrm{CH}-$ type common in fatty acids have been found to exhibit splitting, as in the case of cis-trans-octadecadienoates (absorptions at 10.18 and $10.55 \mu$ ), whereas trans-trans isomers show a single stronger absorption at $10.12 \mu$ [36].

(2) Lunde and Zechmeister [35] showed that, in a series of conjugated diphenylpolyenes, splitting occurred in this region for cis-trans conjugation, but that only shifting of the band to shorter wavelengths occurred in the all-trans isomers. The split bands lay closer together in the higher conjugated systems tested (up to four double bonds) than in the lower ones. These authors suggest that "the two bands may coincide or even exchange places in still higher conjugated systems" [35].

(3) O'Connor and Goldblatt [10] showed that both trans- $\left(\mathrm{C}_{4}\right)$, trans $\left(\mathrm{C}_{6}\right)$ and trans- $\left(\mathrm{C}_{4}\right)$, cis- $\left(\mathrm{C}_{6}\right)$ isomers of the isoprenoid, conjugated triene, allo-ocimene, show splitting, with bands at 10.13 and $10.46 \mu$. These are structures similar to those presumably present in the squalene-sulfur reaction product and in vulcanized rubber.

(4) Lunde and Zechmeister [37] showed that many long-chain carotenoid pigments exhibit only a single absorption near $10.4 \mu$, and that the presence of a central cis double bond adjacent to two $-\mathrm{CH}=$ groups produced a doublet absorbing at 10.35 and $10.47 \mu$.

The above information and the single absorption at $10.4 \mu$ in the present case suggest the presence of some fairly long conjugated systems, absorbing primarily at this wavelength. The work of Lunde and Zechmeister [35] indicates that these systems contain at least one trans double bond. The breadth of the present band should allow for some of these systems to exhibit shifted or split absorptions. No further definition is possible from the position and shape of this band.

An attempt has been made to estimate the percentage of the molecules of squalene-sulfur reaction product containing dienes, trienes, and tetraenes, from their ultraviolet absorbances; and, from this, to estimate the fraction of the band at $10.4 \mu$ due to conjugated systems in the squalene-sulfur reaction product. It must be remembered in the following discussion that large variations in the values of molar absorptivities of given groups, in both the ultraviolet and the infrared, can occur from molecule to molecule. In addition, large variations have been observed, especially in the infrared, in the absorptivities of the same compound reported by different laboratories. These variations reduce the value of the quantitative aspect of these calculations.

The details of the calculation are discussed below:

The ultraviolet molar absorptivities used as reference for 100 percent of molecules absorbing as conjugated dienes and trienes, respectively, were taken from the data for ocimene and for the average of the two isomers of allo-ocimene given in the paper by O'Connor and Goldblatt [10]. A value of 57,500 liters $/$ mole $\mathrm{cm}$ was used for the molar absorptivity of tetraenes, based on the average of data taken from an article by Braude [38] for tetraenes in decatetraenol and for pentaenes in vitamin $\mathrm{A}$. The contribution to the band at $10.4 \mu$ of dienes and trienes, respectively, was estimated from the absorbance of ocimene and the allo-ocimene isomers at $10.4 \mu$, and the data for absorptivities of these compounds were taken from the article by O'Connor and Goldblatt [10]. A value of 34 liters/mole $\mathrm{cm}$ was used for the molar absorptivity of the tetraenes, based on a comparison of band strengths for bands near 3.3 and $10.4 \mu$ in the spectrum of the conjugated tetraene compound designated 4,5-transXVIa by Oroshnik and Mebane [39] and the fact that the absorptivities for the $3.3-\mu$ band for many nonaromatic hydrocarbons seem to average about 45 liters $/$ mole $\mathrm{cm}$ as indicated by the data of O'Connor and Goldblatt [10]. The results of the calculations are given in table 2 . In the absence of knowledge of the molecular weight of the reaction product, the molar absorptivities were calculated by using the molecular weight and density of squalene and the concentration of the squalene part of the molecule in the reaction product. If there is a split absorption in the diene actually present in the squalene-sulfur reaction product, such as is present in the model compound ocimene, a more definite shoulder would be expected at $10.10 \mu$ in the reaction product. If this stronger band of the two is closer to $10.4 \mu$, the effect of the dienes may be increased about threefold, thus allowing conjugation to account for about 59 percent of the band at $10.4 \mu$ in the spectrum of the reaction product. On the same basis, if only that part of the conjugation removed with maleic anhydride is considered (Curve C fig. 5), and using the absorptivity of the stronger band in ocimene as the absorptivity for dienes, about 14 percent of the band at $10.4 \mu$ is accounted for by conjugation. There is some reason to consider only that part of the ultraviolet absorbance removed with maleic anhydride, because of the interference due to sulfur structures discussed in sections 4.4 and $4.5 \mathrm{c}$. This may, however, give low values for dienes because of absorption by maleic anhydride as indicated in section $4.5 \mathrm{c}$.

TABLE 2. Conjugated double bonds in squalene-sulfur reaction product

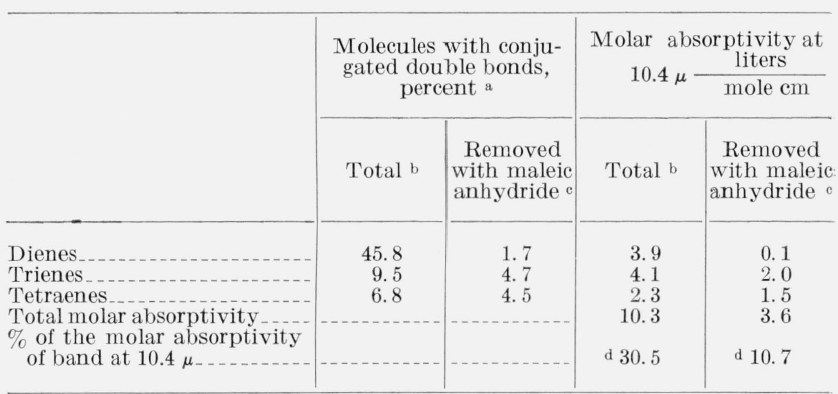

a Indicates percent of molecules containing dienes, trienes, or tetraenes.
b Based on absorbance data from curve A in figure 5 , as given in table 1 .

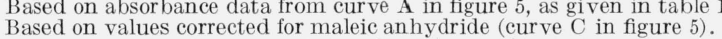

d Based on total molar absorptivity of 33.7 for band at $10.4 \mu$.

The point of this calculation and discussion is to show that, with this much completely reacted sulfur, the material is rather heavily conjugated, and there is good reason to believe that a significant percentage of the band at $10.4 \mu$ is due to conjugated double bonds. There is, of course, room for contribution from isolated trans double bonds and from tetrahydrothiophene structures, especially in the dimer reaction-product where they have been isolated [3], in the squalene-sulfur reaction product where there 
is a shoulder at $10.5-10.6 \mu$, and in unpurified rubber vulcanized with larger proportions of sulfur, where the absorption occurs at longer wavelengths and little absorption at $6.25 \mu$ due to conjugation is noted. Unfortunately, no correlation for isolated trans double bonds is available in the near-infrared at the present time [14].

\section{d. Cther Related Work}

Shipman and Golub [40], in an excellent article, have analyzed the nature of this band, using cispolyisoprene and partially and wholly deuterated cis-polyisoprene to isolate these three effects, and have concluded that the band at $10.4 \mu$ is due largely to conjugated double bonds and tetrahydrothiophenes, with some contribution from isolated trans double bonds.

Apparently, the situation is different in accelerated vulcanizates containing 15 percent of sulfur and 7.5 percent of TMTD, because the conjugation is greatly reduced, as judged by the intensity of the band at $6.25 \mu$, while the band at $10.4 \mu$ is of about the same intensity as in the case of simple sulfur vulcanizates, the reaction presumably being carried nearly to completion in both cases.

Kuz'minskir and Borkova [41], on the basis of infrared studies with ebonite, concluded that the band at $10.4 \mu$ is due to a new double bond that is produced as a secondary reaction of the polysulfides originally formed on reacting rubber with sulfur. They also note the existence of considerable unsaturation remaining in completely vulcanized ebonite as a result of the above sequence. The presence of considerable unsaturation in completely vulcanized ebonite is in agreement with our present and with our earlier findings [2].

Craig, Diller, and Rowe [42] suggested that terminal conjugation, presumably of dienes, could be produced by cleavage of the chain at a radical site on an $\alpha$-methylene carbon atom.

Klauzen and Dogadkin [43] regard the band at $10.4 \mu$ as being due to a trans double bond which, in part, accounts for the difference in physical-mechanical properties between vulcanized and unvulcanized rubber. These authors also consider the band at $6.1 \mu$ to be related to the trans double bond absorbing at $10.4 \mu$.

Except for the absence of a band at $6.25 \mu$, Dale and Artun [44] obtained an infrared spectrum of squalene following dehydrogenation on hydrogenation catalysts that was remarkably similar to that of our squalene-sulfur reaction product. Ultraviolet studies showed that their material, nevertheless, contained conjugated dienes, tetraenes, and hexaenes. The authors considered these changes to have resulted from double-bond migration. This work constitutes further evidence that conjugated systems can absorb near either 6.0 or $6.1 \mu$, since these wavelengths were the only places where bands were observed in the $-\mathrm{C}=\mathrm{C}$ - stretching region.

Dale [45] showed that squalene could be dehydrogenated by $N$-bromosuccinimide to give a wide range of conjugated systems, including those containing $3,5,7,9$, and 11 conjugated double bonds.

The last two studies and the present work are striking examples of how widely different reagents may produce very similar results. They suggest that similar mechanisms may possibly be operating in all three cases.

\subsection{Configuration of Conjugated Double Bonds}

Although rubber is almost entirely cis-polyisoprene, squalene is probably largely trans. In fact, Nicolaides and Laves [46] indicate that it is all-trans. The rather recent near-infrared method of Corish [47] for determining cis and trans configurations in polyisoprenes, when applied to squalene in this laboratory, gave a value of about 43 percent of cis. However, end effects, for example, in the smaller molecule may reduce the quantitative value of this method.

The isomer of allo-ocimene reported by Bateman, Glazebrook, and Moore [3] in their work with dihydromyrcene was predominantly the trans- $\mathrm{C}_{4}, c i s-\mathrm{C}_{6}$ isomer, suggesting that the new double bond required for forming a conjugated triene would be trans. In squalene, if nearly all trans, the introduction of a new trans double bond would yield, usually, the all-trans triene. This does not, however, rule out the presence of cis-trans conjugation in the squalene-sulfur reaction product or the all-trans variety in cis-polyisoprene-sulfur vulcanizates, since various treatments, including the use of heat, have been known to isomerize carotenoid materials [48, 49,50], suggesting the possibility of a mixture of all-cis, cis-trans, and all-trans isomers.

As indicated above, Bateman, Glazebrook, and Moore [3] reported finding the trans, cis isomer of allo-ocimene in the dihydromyrcene-sulfur reaction product. Nevertheless, Chipault and Hawkins [50] indicated that small proportions of sulfur will convert cis into trans conjugated systems at room temperature, as in the case of the conversion of trans-trans-cis $\alpha$-eleostearic acid into the all-trans $\beta$-isomer. Thus, if this latter statement is generally true, it may be that, regardless of whether cis-or trans-polyisoprene is the starting material, conjugated systems in the reaction product will be largely trans.

Some inferences may be attempted from the ultraviolet spectra of the squalene-sulfur reaction product presented here. First, the absorption maxima are remarkably close to those for the presumably all-trans isomers of natural products given by Dale [16], provided that the substitution of cyclohexane or cyclohexane-alcohol for hexane does not affect the wavelengths of the absorption. It has been noted that the first two solvents are interchangeable. Isomers containing cis double bonds absorb, generally, at somewhat shorter wavelengths and tend to lose their distinctive fine structure [49]. Second, the fact that the long-wavelength band for the tetraenes in curve 7 of figure 6 is more intense than the third band suggests the presence of an all-trans or nearly all-trans isomer [16]. Increasing numbers of $c$ is double bonds tend to cause a reversal of this 
band shape [16]. The opposite appears to be true for the trienes pictured in curve 11 of the same figure, but the absorption in the diene region undoubtedly increased the absorption for the short-wavelength band. Furthermore, the work with allo-ocimene would suggest largely, at least, an all-trans triene in the case of squalene. It seems likely that both the trienes and the tetraenes are, for the most part, alltrans. The cis peak [49] present in certain compounds containing cis double bonds in conjugation with trans double bonds cannot be used here, since it is either obscured by the lower polyenes or else would come at too short a wavelength for the instrument to detect. No evidence was found concerning the effect of attached sulfur atoms on the wavelengths of absorptions of conjugated systems, so their presence or absence cannot be indicated.

\section{Conclusions}

Near-infrared studies indicate the absence or near-absence of isolated cis and terminal double bonds in rubber vulcanized either with or without accelerators, as well as in the squalene-sulfur reaction product. The use of purified natural rubber indicates a new absorption near $6.1 \mu$ on reaction with sulfur either with or without accelerators in the rubber system. This new absorption is presumably due either to a double bond with an attached sulfur atom or to conjugated double-bond systems that do not react readily with maleic anhydride. Ultraviolet spectra of chromatographic fractions show that squalene reacted with 15 percent of sulfur contains conjugated trienes and tetraenes and, possibly, dienes, hexaenes, and heptaenes. The conjugated dienes, trienes, and tetraenes are probably present in sufficient proportions to account for for a significant portion of the band at $10.4 \mu$. There is some evidence that the trienes and tetraenes, at least, may be largely all-trans in configuration. Other contributors to the band at $10.4 \mu$ are probably isolated trans double bonds and heterocyclic sulfur structures along the chain. It had previously been shown [2] that the band at $6.25 \mu$, attributed to conjugation, first appears with about 5 percent of sulfur and that the band at $10.4 \mu$ first appears at about 2 percent of sulfur. It may, therefore, be assumed that the structures attributed to these bands are formed at considerably smaller concentrations of sulfur than those used here. Variations in the relative proportions of the various conjugated systems with sulfur content are to be expected.

\section{References}

[1] N. Sheppard and G. B. B. M. Sutherland, J. Chem. Soc. 1947, 1699; Rubber Chem. Technol. 21, 799 (1948).

[2] F. J. Linnig and J. E. Stewart, J. Res. NBS 60, 9 (1958) ; Rubber Chem. Technol. 31, 719 (1958).

[3] L. Bateman, R. W. Glazebrook, and C. G. Moore, J. Chem. Soc. 1958, 2846; Rubber Chem. Technol. 31, 1065 (1958)

[4] E. T. McDonel, J. R. Shelton, J. Chem. Eng. Data 4, 360 (1959).
[5] J. L. Bolland and Geoffrey Gee, Trans. Faraday Soc. 42, 244 (1946); Rubber Chem. Technol. 20, 617 (1947).

[6] T. J. Murphy, W. S. Clabaugh, and R. Gilchrist, J. Res. NBS 64A, (Phys. and Chem.) 355 (1960).

[7] Association of Official Agricultural Chemists, Official Methods of Analysis of the Association of Official Agricultural Chemists, 9th edition, p. 654 (Association of Official Agricultural Chemists, Washington, D.C., 1960).

[8] L. J. Bellamy, The Infra-red Spectra of Complex Molecules, p. 36 (John Wiley \& Sons, Inc., New York, N.Y., 1958).

[9] Y. Mikawa, Bull. Chem. Soc. Japan 29, 110 (1956).

[10] R. T. O'Connor and L. A. Goldblatt, Anal. Chem. 26, 1726 (1954).

[11] G. F. Bloomfield, J. Chem. Soc. 194\%, 1546.

[12] L. C. Bateman, R. W. Glazebrook, C. G. Moore, and R. W. Saville, Proceedings of the Third Rubber Technology Conference p. 298 (W. Heffer and Sons, Limited, Cambridge, London, 1954); Rubber Chem. Technol. 30, 397 (1957).

[13] R. W. Glazebrook and R. W. Saville, J. Chem. Soc. 1954, 2094; Rubber Chem. Technol. 28, 109 (1955).

[14] R. F. Goddu and D. A. Delker, Anal. Chem. 32, 140 (1960).

[15A] D. W. Fraga, J. Polymer Sci. 41, 522 (1959); Rubber Chem. Technol. 33, 982 (1960).

[15B] J. L. Binder, J. Polymer Sci. 1, 37 (1963).

[16] J. Dale, Acta Chem. Scand. 8, 1235 (1954).

[17] H. P. Koch, J. Chem. Soc. (London) 1949, 387.

[18] H. P. Koch, ibid, 1949, 394.

[19] R. E. Davis, J. Org. Chem. 23, 1380 (1958).

[20] Y. Minoura, J. Inst. Polytech., Osaka City University, Ser. C 5, 206 (1956); Rubber Chem. Technol. 31, 808 (1958).

[21] J. E. Baer and M. Carmack, J. Am. Chem. Soc. r1, 1215 (1949).

[22] A. F. Postovskaya, M. A. Salimov, and A. S. Kuzminskiŭ, Dokl. Akad. Nauk SSSR 114, 586 (1957); Rubber Chem. Technol. 31, 747 (1958)

[23] B. A. Dogadkin and N. N. Pavlov, Vysokomolekul. Soedin. 3, 613 (1961); Rubber Chem. Technol. 36, 262 (1963).

[24] J. K. Bartlett and D. A. Skoog, Anal. Chem. 26, 1008 (1954).

[25] C. G. Moore and M. Porter, Paper presented at Conference Internationale sur le Caoutchouc, Paris, May 14-18, 1962

[26] L. F. Fieser and M. Fieser, Advanced Organic Chemistry, p. 206 (Reinhold Publishing Corp., New York, 1961).

[27] G. Dupont, R. Dulou, V. Desreux, and R. Picoux, Bull. Soc. Chim. France [5] 5, 322 (1938).

[28] L. H. Flett and W. H. Gardner, Maleic Anhydride Derivatives-Reactions of the Double Bond, p. 6, (John Wiley \& Sons, Inc., New York, N.Y., 1952).

[29] C. Pinnazi, R. Cheritat, and R. Pautrat, Paper presented at Conference Internationale sur le Caoutchouc, Paris, May 14-18, 1962.

30] D. Craig, B. F. Goodrich, Research Center (private communication).

[31] G. F. Bloomfield, J. Polymer Sci. 1, 312 (1946); Rubber Chem. 4 Technol. 20, 360 (1947).

[32] G. F. Bloomfield, J. Chem. Soc. 1947, 1547; Rubber Chem. Technol. 22, 348 (1949).

[33] G. F. Bloomfield and R. F. Naylor, Proc. XIth Intern. Congr. Pure Appl. Chem., Volume II, p. 7, Organic Chem., Biochem. London, England, 1947.

[34] F. J. Linnig, E. J. Parks, and L. A. Wall, J. Res. NBS 65A, 79 (1961).

[35] K. Lunde and L. Zechmeister, Acta Chem. Scand. 8, 1421 (1954).

[36] J. E. Jackson, R. F. Paschke, W. Tolberg, H. N. Boyd, and D. H. Wheeler, J. Am. Oil Chemists' Soc. 29, 239 (1952).

[37] K. Lunde and L. Zechmeister, J. Am. Chem. Soc. $\boldsymbol{y}$, 1647 (1955).

[38] E. A. Braude, Ann. Rept. Progr. Chem., (Chem. Soc. London) 42, 105 (1945). 
[39] W. Oroshnik and A. D. Mebane, J. Am. Chem. Soc. z6, 5719 (1954).

[40] J. J. Shipman and M. A. Golub, J. Polymer Sci. 58, 1063 (1962).

[41] A. S. Kuz'minskiľ and L. V. Borkova, Zh. Prikl. Khim. 31, 648 (1958) ; J. Appl. Chem. USSR (English Trans.) 31, 635 (1958).

[42] D. Craig, D. Diller, and E. H. Rowe, J. Polymer Sci. 28, 435 (1958).

[43] N. A. Klauzen, and B. A. Dogadkin, Zavodsk. Lab. 28, 438 (1962).

[44] J. Dale and T. Artun, Acta Chem. Scand. 10, 439 (1956).
[45] J. Dale, Arch. Biochem. Biophys. 41, 475 (1952).

[46] N. Nicolaides and F. Laves, J. Am. Chem. Soc. $\boldsymbol{7 6}, 2596$ (1954).

[47] P. J. Corish, Spectrochim. Acta 1959, 598 Rubber; Chem. Technol. 33, 975 (1960).

[48] P. Karrer and E. Jucker, Carotenoids, p. 38 (Elsevier Publishing Co., Inc., New York, 1950).

[49] L. Zechmeister, Experientia 10, 1 (1954).

[50] J. R. Chipault and J. M. Hawkins, J. Am. Oil Chemists' Soc. 3\%, 176 (1960).

(Paper 68A5-296) 